DOI: $10.1515 /$ hssr -2015-0018

\title{
Towards a Semiotics of the "Loving Light": the Transmodern Turn
}

Traian-Dinorel D. Stănciulescu*

“Alexandru Ioan Cuza" University of Iași \& National Institute of Inventics of Iasi, Romania

\begin{abstract}
The core of what we call transmodern turn is sustained (after decades of dominance of post-modern epistemology) by the shaping of an ontological model of the "Essentials Unity", in which Human Being, the World and God should be in a non-conflictual relationship of togetherness, by a resonant / holographic mechanism of light. (Re)cognizing that the world-object and metalanguage have an objective interface, religion, philosophy and modern (social) sciences harmonise their specific assertions through a semiotics of the "loving light" capable of proving that: syntactically, the world is governed by unifying patterns which contain the semantic meanings of an objective human "language of light"; from a pragmatic point of view, biophotonically assumed, the fundamental values of human being / history can be traced in the ontologic (natural - cultural) act of signification which foregrounds the becoming of universal resonance into love.
\end{abstract}

Key-words

Transmodernity, Synergy, Unification, Signs of light, Resonating love.

"There is nothing new under the Sun": here is a statement that does not need argumentation. Because it is already a truism to say that everything that was will be again, and that everything that is had already been. But at

\footnotetext{
* Faculty of Philosophy, "Alexandru Ioan Cuza" University of Iasi, 11 Carol I Ave, 700 505, Iași, Romania; tdstanciu@yahoo.com
} 
Traian-Dinorel Stănciulescu, Towards a Semiotics of the "Loving Light ...

HSS, vol. IV, no. 2 (2015): 119-134

the same time the paradoxes of existence permit us naturally to say that: "Everything is new under the Sun." Because at every moment of its existence "something" changes its state and the passing of time allows us to:

- look on the world through the eye of its constancy, of its perenniality, which allows everything to be recognizable and recallable;

- $\quad$ see with different eyes the constant change of forms, of their becoming, along the path of their appearance and disappearance.

This is how the world, being itself conceived / structured holographically by the Great Creator, finally allows the human being - a measure of "the image and likeness" - to have a holographic view, able to grasp at the same time the constancy and the ephemerality, the phenomenon and the essence, the continuity and the discontinuity. With a certain effort, the "rational eye" can see what intuition whispered to it long ago: perfect forms were designed by the divinity over the clay of matter in order to engender the infinity of forms by which the world itself began to evolve. For human beings, all these forms have become the "language-object" of a collective symbolical assumption, supposing a general type of "metalanguage".

Here we have, all together, creationism and evolutionism, spirituality and science, harmonically assumed in the frame of two paradigms - among many others - of the Third Millennium: the CONNECTION SYNERGY and the SYMBOLICAL COMMUNION. To develop the premises of these two paradigms is the purpose of the present article: to elaborate a strategy for human harmonization by using the unifying force of the symbolical (verbal) language, the LANGUAGE OF LIGHT, specifically (Stanciulescu, $2003 \mathrm{a}, \mathrm{b}$ ).

Finally, let us remember that such a purpose - able to connect the past and the future knowledge - also represents the main aspiration of the new Aquarian Era, of the new TRANSMODERN TURN: to recuperate unity from diversity... 
Traian-Dinorel Stănciulescu, Towards a Semiotics of the "Loving Light ...

HSS, vol. IV, no. 2 (2015): 119-134

\section{1. (Re)Understanding the meanings}

If there is no LOVE, there is nothing! (Marin Preda)

Or, mutatis mutandis:

If there is no CONNECTION, there is nothing!

It is already a truism to say that man is a symbolically interactive being, as Herbert Mead has argued, a social / cultural being having Aristotle’s zoon politikon as an essential characteristic. But, it is less common to assert that - before all - it was the symbolical / semiotic function which defined humanity's essential feature: the capacity to reflect / assume consciously his / her ESSENTIAL UNITY and CONNECTION, with her / himself and with her / his FELLOW, with COSMOS and GOD. In other terms, the primary quality of homo significans defined the later one of bomo sapiens.

In this context, it is natural that we "should look until we can see" as the sculptor Constantin Brâncusi used to urge us - in order to credit the statement of the mathematician David Hilbert: "In the beginning was the SYMBOL / SIGN...." (Stanciulescu 2005). Where does the ever more present and nuanced interest in rediscovering, in the history of human thinking, this integrative doctrine of symbols-signs, of the multiple forms of representing the world, come from? Have modern human beings begun to understand that they themselves cannot exist as spiritualized beings outside the symbol-sign?

According to Ferdinand de Saussure's definition, any sign / symbol supposes a signifier / symbolizer form (a perceptible support / form) and a signified / symbolized (an imperceptible information / content). Bearing this definition in mind, two possible interpretations: an ontological and a gnoseological one, are available to answer the questions above.

(1) Ontologically, human beings have understood, in time, that it is through the very SYMBOL that they can complementarily define themselves - in the frame of the "ESENTIAL UNITY" that has been structurally and functionally established between buman being and macro / microcosm, on the one hand, between human being and God, on the other hand (Stanciulescu, 2003b) - as: 
Traian-Dinorel Stănciulescu, Towards a Semiotics of the "Loving Light ...

HSS, vol. IV, no. 2 (2015): 119-134

- an expression of the divine creation, for which - according to Charles Peirce - LOVE is an integrative measure: the WORD-LIGHT;

- a measure of the action of cosmic becoming laws, with RESONANCE / love as the harmonizing principle of certain "magic" symbols-signs;

- an outcome of the social-historical progress, essentially based on the COMMUNION / COMMUNICATION with fellow beings by means of certain more or less "conventional symbols / signs".

(2) Gnoseologically, if the FORM - as an expression of a "wrapped" SYMBOL / SIGN - can be placed before all beginnings as an energoinformational matrix of the world that is about to "unwrap", as naturally believed the physicist David Bohm, then SEMIOTICS / HERMENEUTICS / COMMUNICOLOGY, the science(s) of defining and interpreting the genesis and functions of the symbols-signs ("symbolic forms") and of communicating with their help, should have been born before any other science. How do representatives of so many other disciplines, many of whom have not even heard of semiotics, respond to such an epistemological challenge?

In a synthetic / implicit manner, the answer is offered by Ernst Cassirer's "Philosophy of Symbolic Forms" (1925, 1925, 1929), which perfectly connects ontology and epistemology by suggesting the distinction between symbol and sign, as follows:

- by its strong iconicity - characterized by the energetic-substantial connection between the symbolizer and the referential - the symbol is more attached to nature;

- by its essential conventionalism - determined by the energetic and informational arbitrariety between the signifier and the named object - the sign is connected especially to culture.

Such a theoretical understanding is losing its roots with the growth of human knowledge, oscillating between symbol and sign, nature and culture, physis and thesis, between archaic and modern, and having as an archetypal problem the genesis of the world itself.

Historically speaking, the intuition of the PRIMORDIALITY OF THE SYMBOL-SIGN in relation to the cosmic world is a feature of the mythical-symbolical thinking privileged by Ernst Cassirer in his essential research. The ex nibilo model of the creation of the world associates, to 
Traian-Dinorel Stănciulescu, Towards a Semiotics of the "Loving Light ...

HSS, vol. IV, no. 2 (2015): 119-134

the birth act of the world, a series of non-verbal symbols that the demiurge could have used for this purpose: shout, murmur, laughter, crying, whistling, gesture, dance, music or, finally, articulate words. The use of the symbol for the purpose of taking the world out of chaos is a creation of a magic type because "the Creative God does nothing else than a wizard does by his spells and charms" (Daniel 1985: 291).

The universality of this kind of cosmogony is due to the fact that almost all peoples of the world relate the beginnings of their knowledge to the practice of magic, within which "the power of the symbol" plays an essential part. Numerous relevant examples can be mentioned for each of the types of symbols (symbolizers) that initiated the creative act. The motif of the creation of the world by the symbol-word must not always be identified with an ex nibilo creation; by expressing the symbol of the sign (verbal or non-verbal), the spirit "gives, at the same time, substance (life) and form [symbolizer and symbolized, our note, TDS] by what the word signifies and determines [the referential, our note, TDS]". In other words, in this stage of the cosmic creation, the myth gives priority to the physical (life-symbolizer) and not to the intentional (of the symbolizing act).

In archaic cosmogonies the symbol-light can hold two functions: as initiator of the cosmic semiosis, as "symbol-cause"; or as outcome of a first semiosis ("symbol-effect") and, respectively, of refining instrument of the cosmic creation within a second semiosis (which comprises the appearance of humanity and of the symbol / the human word). A few semiotic remarks can be associated to this characteristic feature of the "second" word.

"The word" of this stage of cosmogony is an outcome of the "blend of reason and speech", of the manifestation of a latent content-signified by an exterior signifier-physical substratum. Such a "word", displayed by means of a concrete cosmogenesis (checking the existence of land on the bottom of the waters, for example) or of an abstract cosmogenesis (the designing by the god of the project of the creation of the world, for example) becomes the instrument of certain real achievements like the separation of waters from land, the creation of light, etc. It thus defines the background of the paradoxical meeting between the infinite and the finite, the eternal and the transient, by uniting the "profound" signified 
(project, desire of divinity) with the physical, "surface" signifier, detached and at the same time anchored in the ephemerality of the world.

Identifying the symbol-sign with the word - in its quality as articulate sign, according to the biblical sentence "In the beginning was the word" - is the characteristic of a more refined thinking, already close to "modernity". All subsequent attempts to give a theoretical definition to the SIGN will be based on this assumption, for which the hierarchies of the "COMPLEX LIGHT"1 represent the measure. The whole of these attempts is the very basis for the birth of semiotics itself.

Developed around the idea that semiotics is an integrative doctrine of the "symbols / signs of all times", which have benefitted from the recent contributions of scientific knowledge (from cosmology to cybernetics or biophotonics, for example), the present work can be considered as another kind of INTRODUCTION TO SEMIOTICS: an unconventional one. It takes the form of a synthesis coherently organized around the idea of SYMBOL_CREATIVE SIGN / WORDLIGHT and of its power to harmonise, analysed in one of its possible positions:

- at the level of cosmic reality, by examining the relation between the world-engendering symbol / sign and the insufficiently known mechanisms that activated its power (Stanciulescu, 1995);

- at the level of human reality, by highlighting the historical modes from the archaic to the modern ones - which accompanied the transformation of the immanent (natural) power of the symbol / sign into a predominantly conventional (cultural) one (Stanciulescu, 2005).

This very junction between the world of cosmos and the human world was the universe of understanding in which Ernst Cassirer projected his semiotic "philosophy of symbolic forms", arguing - along the same way followed later by Sapir-Whorf - that the symbolical language reflects the presence of cosmos in the human being and of the human being in cosmos. In other words: an understanding determined by the need of the buman being to (re)define itself as a cosmic and, at the same time, historical being, which today only poetry and music - synergically coupled by LUXONICS, the integrative science of LIGHT \& SOUND - have the force to recover and reconfigure symbolically in full. 
Traian-Dinorel Stănciulescu, Towards a Semiotics of the "Loving Light ...

HSS, vol. IV, no. 2 (2015): 119-134

\section{The "Connection Synergy", a measure of the generative love}

Following up closely on what we have stated above, we can see here how the concept of CONNECTION which, at first sight, seems to come from the sphere of scientific / technical activities, can acquire the symbolical value of spiritual archetypes. In a broad acceptation, connection should also be understood as LOVE, that is resonance or coherence, affinity, compatibility or empathy, etc. It is a truism to say that if at a certain moment, by a certain act of "divine intervention", LIGHT - assumed as structural / structuring information - had not emerged from the primordial chaos / dark, the cosmos / the world could not have been born. In other words, what made this genesis possible was the very appearance of "the capacity to love", to connect two particles, two atoms or molecules, two structures or two living entities, etc. in order to generate more and more complex systems. That is why Charles S. Peirce could justly state that the most powerful sign of the existence of God in the world is LOVE.

While this availability of the physical or biological nature is predominantly informational, the connection proper, the attraction and coherent coupling of two physical or bio-psychical entities in a whole is of an energetic nature, involving a certain "coupling force", measuring the degree of attraction / rejection, of complementarity between the two systems, between their more or less different states. This is the force that makes it possible, for example, to maintain the most refined connection in the world: the one between the wave and the corpuscle, between the soul and the body, between the divine and the human spirit.

The universal INTEGRATIVE LOVE, RESONANCE and CONNECTION too...

So, it is in this universal "coupling force" of LOVE that the phrase CONNECTION SYNERGY2 also finds its origin. The sphere of this concept is maximal because it covers, by the actions it involves:

- the metalanguage of synergy, understood as the cooperation of all the parts of a certain system - of the world itself - which generate a more powerful effect than the sum of the parts;

- the language object of the four elements, of all the "reality levels" of the macro- and micro-cosmos - substantial and undulatory, energetical and 
informational - manifested at the (meta)physical and biological, psychical and social levels, along the spiral that mounts from nature to culture, from human beings to God.

In this context, we could appreciate that SYNERGY - represented in the frame of an "essential square" - defines both the STRUCTURAL and FUNCTIONAL coherent / connected manifestations of the four archetypal elements of the old and modern cosmogony:

$\rightarrow$ fire $/$ field $=$ the potentiality of the resonance (creative dynamism);

$\rightarrow$ air $/$ information $=$ the potentiality of the (perfect created) forms;

$\rightarrow$ water / energy = real manifested dynamism (creative processes);

$\rightarrow$ earth $/$ substance $=$ real manifested (imperfect / evolved) forms.

As a synthesis, at the intersection point of the diagonals of the "essential square" (Figure 1) - it also determines a NEW STRUCTURAL ELEMENT, and a NEW FUNCTION, the fifth one. So:

- Structurally, this integrative "cosmogonic element" could be symbolically identified with the "UNCREATED LIGHT," in spiritual terms, or with the "ETHER" of the old alchemists.

- Functionally, this integrative element has its own function, expressed as the power of the FIFTH FORCE, connecting the potentiality and reality, the dynamism and form by the force of CREATIVE LOVE.

\section{ETHER \& SYNERGY}

Non-manifested (potential) world

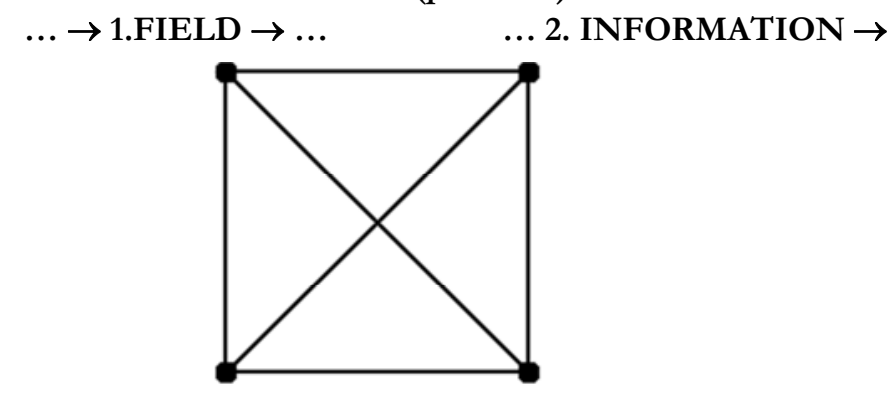
$\rightarrow$ 3. ENERGY $\rightarrow$ ... 4. SUBSTANCE $\rightarrow$
... LOVE (resonance / communion)
Manifested (real) world

Figure 1. The "essential square": structural and functional world connecting synergy 
By looking at the above mentioned relations we could reformulate / postulate - intuitively only in this context ${ }^{3}$ - some (meta)physical hypotheses of the "ESSENTIAL UNITY" THEORY (Stanciulescu, 2003b) such as:

- The central point of the square defines the "Zero Point" of everything, the CONNECTION SYNERGY's unfolded potential, DIVINITY, LOVE, and LIGHT-WORD potentiality. Consequently, the WORLD is becoming a coherent result of the CONNECTION SYNERGY.

- The circular connection of the square's elements (from 0 to 1 $\rightarrow \ldots 4$ ) suggests that: (a) ontologically, the world is created from invisible to visible, from field to substance; (b) gnoseologically, the reality of the world is recuperated by human knowledge from visible to invisible, from the physical to the metaphysical reality.

- The horizontal relations of the square allow the following connections: (a) the field mainly preserves information; (b) the substance essentially preserves energy.

- The vertical connections of the square suggest that: (a) the properties of the field activate a specific energy; (b) the properties of the information activate substantial forms.

- The square's diagonals show that the main couples / dualities of the world are the following: (a) the partial visible corpuscle-wave (substancefield) couple that quantum physics discovered (Bohr, Louis de Broglie); (b) the invisible energy-information (ergon-biton) couple (Constantinescu, Stanciulescu, 1993).

These last connections could be further linked to the properties of the COMPLEX LIGHT, as follows:

- The field defines light as a vibratile / ondulatory reality, conforming to the ancient (Rig-Veda) and modern (quantum physics) principle: "Everything is vibration / wave" (Rig-Veda / Max Bohr).

- The information could be associated with the light capacity to generate forms, by attribute of spin, frequency and especially of the magnetic component of its waves.

- The energy could be associated with the powerful action of the electric component of the light-wave. 
Traian-Dinorel Stănciulescu, Towards a Semiotics of the "Loving Light ...

HSS, vol. IV, no. 2 (2015): 119-134

- The substance is represented by the very small mass of photons, which was already discovered by scientists, by permitting experiments such as the (de)materialization of the macro- and micro-bodies, the "frozen laser light", etc.

Starting from all the considerations above, in generalized terms, we could assert that: the substance (mass quantity) and the energy (the dynamic power), information (frequency, amplitude, spin) and field (wave type of vibration, speed etc.) of a certain closed system are permanently and equivalently transforming into the frame of another system, by conserving some essential properties. Or, in the already known common terms: nothing changes, everything is transformed only.

This assertion represents a new and completed expression of the GENERAL LAW OF MATTER TRANSFORMATION, where matter means the synergy of substance, energy, information, and field.

According to the above arguments, Einstein's assertion that EVERYTHING IS LIGHT (a form of the "complex light", namely) in different degrees of concentration, seems to be perfectly true, once again. And, implicitly, his FORMULA: $\mathbf{E}=\mathbf{m c}^{\mathbf{2}}$, synergically connects the presence of the four elements, objectively associated to light, as follow:

- $\boldsymbol{m}=$ mass / substance, expressing the quantity of photons present in the frame of the system under consideration;

- $\boldsymbol{E}=$ energy $=\boldsymbol{h} \boldsymbol{v}$, namely: Planck's constant $/$ coefficient of the energy emitted by photons, multiplied with:

- Information = frequency of the light system, responsible not only for the energy of the system, but also for its morphology / dimensional form (the result - form coupled with energy - depends on the "colour" type / frequency of the system, in the case of white light, in particular, by the specific level of the electromagnetic spectrum, under- or overluminous respectively);

- $\boldsymbol{c}^{2}=$ light speed, is connected to one of the essential properties of light: movement efficiency, which is implicitly determined by the specific medium of propagation, the field itself (magnetic or electric, gravitational, etc.). 
Traian-Dinorel Stănciulescu, Towards a Semiotics of the "Loving Light ...

HSS, vol. IV, no. 2 (2015): 119-134

This formula - indeed one of the most integrative formula ever known - justifies the general law of matter transformation, but only at the level of the macrocosmic light, moving in vacuum at a speed of 300,000 $\mathrm{km} /$ second. But Einstein's formula should be completed / specifically adapted to the situation of all the complex light systems, on the one hand, and to the case of open systems (permanently exchanging substance and energy, information and fields among them), from cosmic systems to human beings (body and soul, mind and spirit), on the other hand. We refer precisely to a connection between cosmic light and human COMPLEX LIGHT.

In this perspective, we should take into consideration the law of matter transformation in order to better understand the becoming of the human being, of human DESTINY namely.

\section{By way of conclusion}

Upon analysing the hierarchies of human becoming / destiny, we discover that the life of human beings can be essentially influenced by their belonging to / dependence on "light's hierarchies", on the "sky" to which their "birth chart" belongs, on their parents' genes and on the psycho-social vibration of their birth and life environment, on cosmic and natural factors, and on the material and technical factors that influence their personality or health, etc.

- It is by some of these "destiny factors", which are determined by birth, that human beings are compelled to connect, most of the time, unconsciously. On no account will one be able to change, in the course of his or her life, the quality of being both "star dust" and "a bit of divinity", as Carl Sagan said, of having been born under a certain sign of the zodiac, of having a certain genetic history, etc. Therefore, human beings must only connect / adapt to this category of objective factors, which are impossible to transform (because they are outside the human power of action, on the one hand, and because history cannot be modified, on the other hand). Depending on the goals pursued in life, such a connection / adaptation (optimally) requires human beings to spend a certain quantity of CONNECTION SYNERGY. The smaller this quantity, the more human beings are able to redistribute the 
Traian-Dinorel Stănciulescu, Towards a Semiotics of the "Loving Light ...

HSS, vol. IV, no. 2 (2015): 119-134

conserved substance and energy, information and vibratile field to other purposes, of practical or spiritual meanings.

Diminishing this synergy, which human beings have the "duty" to pay to their cosmic and divine existence, involves one thing: acting with full knowledge, in agreement / harmony / love with the imperative law. For example, it would be absurd for a human being to spend its vital energy trying to be able to never die, because it is known that nobody can escape "the imperative of death".

- We must also say that, besides this category of "transpersonal" determinations, whose action can be most known and accepted, there is a series of other "local" determinations that human beings can influence directly: part of the forms of the cosmic-geographic space (earth, water, air, fire) and of the artificial one (the objects defining the daily life environment, the architecture, the furniture and the clothing), life options (food and medication), work options (activity and rest), etc. Human beings can act upon these categories of determinations in such a way that, from the very beginning, by a correct (ecological) attitude and an appropriate design (Biophotonic Synergy Design) (Poenaru, Stanciulescu, 2006), they can require from it only a minimum consumption of ADAPTATION INFORMATION.

Intuitively, the distinction formulated between the terms "connection" and "adaptation" could be assumed in the following terms:

- the former must be correlated especially to the objective "symbolizer energy", which makes possible the physical interaction of two systems, natural or human, able to be preserved in a certain measure by the implicated human being; for example, by their physical resonance, by their frequency modulation, intensity and timber, human voice sounds (speech, music etc.) could objectively influence the body health condition;

- the latter must be associated preponderantly to the subjective "symbolized information", which makes possible the spiritual interaction of (human) systems; this information can be modified by interested / capable human beings; for example, the semantic content of one's speech also determines human health, by influencing positive or negative thinking, and implicitly a strong biophysical activity of the body; such 
Traian-Dinorel Stănciulescu, Towards a Semiotics of the "Loving Light ...

HSS, vol. IV, no. 2 (2015): 119-134

types of effect can be controlled / adapted by human volition to obtain certain expected results.

Consequently, the complementarity between the "connection energy" and "adaptation information" is very clear, by defining the two dimensions of a symbol / sign. How can we account for our preference to mention the "connection energy" only in the title of the current article? Well, in the context of symbolic languages optimization / harmonization, which interests us here, energy is twice implicated:

- on the one hand, for the modification of the substratum of the symbolizer / signifier form, by consuming a certain quantity of natural (physical) energy;

- on the other hand, for the generation of a new informational sense of the symbolized / signified content, by implying a mental consumption of (bio-psychical) energy.

It is interesting to notice that such a metaphysical analysis is able to generate a physical hypothesis, namely: that the universal law of the substance and energy transformation and conservation must be doubled by a similar law of the information and field transformation and conservation by bolographic resonance (Stanciulescu, 2011). As we have already stated, both types of laws suppose the possibility of a "harmonious connection" between the implied systems, being accomplished by the force of not only a symbolical universal integrative law:

- the law of matter "connection synergy", symbolically assumed also as:

- the law of conserving and transforming the "Light of Love".

These laws concern the main path of increasing the frequency of the buman being (the frequency of his / her body, soul, mind, biophotonically explained) elevating his / her "light of love" towards some still ignored hierarchies of "Living Light".

Hierarchies which certainly our colleague and friend - Tiberiu Brailean - who has ascended to higher spheres, already knows. 
Traian-Dinorel Stănciulescu, Towards a Semiotics of the "Loving Light ...

HSS, vol. IV, no. 2 (2015): 119-134

\section{References}

Cassirer, Ernst. The Philosophy of Symbolic Forms. 3 vols. Vol. 1, Language (1953); vol. 2, Mythic Thought (1955); vol. 3, The Phenomenology of Knowledge (1957). Trans. Ralph Manheim. New Haven: Yale University Press.

Constantinescu, Paul, Stanciulescu, Traian D., "Resonance as a Principle of Universal Creativity. Photonic (Quantic) Hypothesis of InformationEnergy", in Revista de Inventica, Iasi, no. 12, 1993, 18-25.

Daniel, Constantin. Cultura spirituala a Egiptului antic (Spiritual Culture in Ancient Egypt). Bucuresti: Cartea Româneasca, 1985.

Poenaru, Aritia, Stanciulescu, Traian D. Vestimentatia luminii. Armonizarea sanatatii prin "design synergetic" (Clothing of Light. Health's Harmonization by "Synergetic Design"), Iasi: Performantica, 2006.

Stanciulescu, Traian D. Miturile creatiei. Lecturi semiotice (Creation Myths: Semiotic Lectures), Performantica, 1995.

Stanciulescu, Traian D. Semiotics of Light. An Integrative Approach to Human Archetypal roots, second edition, Iasi-Geneva: Cristal-Concept, 2003 a.

Stanciulescu, Traian D. Signs of Light. A Biophotonic Approach to Human (Meta)Physical Fundamentals. second edition, Iasi-Geneva: Cristal-Concept, $2003 \mathrm{~b}$

Stanciulescu, Traian D. La inceput a fost semnul: o alta introducere in semiotica (In the Beginning There Was the Sign: Another Introduction to Semiotics), Iasi: Performantica, 2005.

Stanciulescu, Traian D. Exogenous Homeopathy. A Synergetic Therapy: the Explanatory Contribution of Biophotonics, Iasi: Performantica, 2011.

1 The phrase "COMPLEX LIGHT" involves the assembly of all possible manifestations of LIGHT, starting from the electromagnetic radiations spectrum to the $2,7 \mathrm{~K}$ cosmic remnant radiation of the Big-Bang, from the bioluminescence of human, animal, plants living systems to the presumptive noesic "Hierarchies of Light" and, finally, to the highest "Uncreated Light".

${ }^{2}$ The content of this concept has enriched over time; the principle of the conservation of substance and energy has been intuitively developed. Initially we used the alternative "CONNECTION ENERGY" in a meeting of the International Communicology Institute (Illinois, USA) organized in Aalborg / Skagen (Denmark) (Stănciulescu, 2006), in order to suggest that the energy consumed by human connection with the surrounding frame could be both "prophylactically and therapeutically" minimalized. After that, only, did we realise that the same considerations are necessary for substance, information 
and field. At that moment, the intuition of a general law of conservation of matter (of field and information, of energy and substance) was already conceived, under the integrative force of the "CONNECTION SYNERGY" concept.

${ }^{3}$ The assertions that follow represent only the first step of a very serious effort to prove, in the terms of scientific (physic-mathematical) language, the truth of some older and latent intuitions. The time of their clear expression seems to be near.

IT WAS TO BE, EXACTLY LIKE THAT...

To my peacefully ascended friend, TIBERIU BRĂILEAN.

I am wondering

at the dawn of my awakening

if the Music of Spheres

is still as round

as the sages could hear it resound,

when talking about the Terrestrial Waves

already pursuing each other among the stars...

I am still wondering

if the Symphony of the Human-Earth

can also be heard by the Celestial Listeners,

if, in the very beginning,

the creators from High Up in the Skies

made our Soul-Tympanum,

so delicate, so resonant...

I am really wondering

if the Godhead beyond the Heights,

without a wind-caressed body,

without a breathed-in breath,

wished us to be as He only knows how,

to His image and likeness, turning us into Flesh Alive

as a smile of the chosen path of life,

as a poem for which 
Traian-Dinorel Stănciulescu, Towards a Semiotics of the "Loving Light ...

HSS, vol. IV, no. 2 (2015): 119-134

there is not a living poet able to write it, nor to marvel at the wondrous beauty

of Coming unto Being.

I am finally wondering, again, much more confused than ever:

how should it sound now, the Bow of Thanks, given to those who made us into such noble beings, without ever living the privilege of Human Destiny?

Traian-Dinorel D. Stănciulescu Iasi, 9th of March, 2015 